IGCS20_1219

\section{EFFECT OF OLAPARIB TREATMENT ON CELL CYCLE, SENESCENCE AND CELL DEATH IN OVARIAN CANCER CELLS}

${ }^{1} \mathrm{P}$ Tudrej* ${ }^{*}{ }^{2} \mathrm{M}$ Głowala-Kosińska, ${ }^{3} \mathrm{D}$ Sojka, ${ }^{4} \mathrm{AJ}$ Cortez, ${ }^{3} \mathrm{KM}$ Lisowska. ${ }^{1}$ Department of Genetic and Molecular Diagnostics of Cancer, Maria Skłodowska-Curie National Research Institute of Oncology, Poland; '2Department of Bone Marrow Transplantation and Hematology-Oncology, Maria Skłodowska-Curie National Research Institute of Oncology, Poland; ${ }^{3}$ Center for Translational Research and Molecular Biology of Cancer, Maria Skłodowska-Curie National Research Institute of Oncology, Poland; ${ }^{4}$ Department of Biostatistics and Bioinformatics, Maria Skłodowska-Curie National Research Institute of Oncology, Poland

\subsection{6/ijgc-2020-IGCS. 187}

Objectives High-grade serous ovarian cancer (HGSOC) is the most frequent and deadly histological type of ovarian cancer. Targeted treatment approaches, such as PARP inhibitors, have brought significant improvement in management of this disease. Here we analyzed molecular mechanisms of olaparib influence on ovarian cancer cells.

Methods We used our newly established HGSOC cell line OVPA8 (ECACC \#1906260 ${ }^{1}$ ) and three other lines (A2780, SKOV3, KURAMOCHI). BRCA1/2 mutations were analyzed by Sanger sequencing and NGS. Cells were treated by olaparib (AZD2281). Metabolic activity and viability were assessed using AlamarBlue and crystal violet assay, respectively. Half maximal inhibitory concentrations (IC50) were determined using GraphPadPrism software. Cell death and cell-cycle distribution were analyzed by flow cytometry.

Results NGS analysis revealed BRCA1 pathological mutation c.3700_3704delGTAAA (p.Val1234Glnf) and loss of heterozygosity in BRCA2 in OVPA8. BRCA2 pathological mutation c.6952C $>$ T (p.Arg2318Ter) was described in KURAMOCHI, BRCA2 mutations of unknown significance in A2780 (c. T8195G, p.L2732X) and SKOV3 (c.7364A>G, p.H2455R). ${ }^{2}$ The IC50 values were $3.99 \mu \mathrm{M}, 5.13 \mu \mathrm{M}, 6.77 \mu \mathrm{M}$ and $25.6 \mu \mathrm{M}$ for OVPA8, A2780, KURAMOCHI and SKOV3, respectively. Olaparib treatment resulted in $G 2 / M$ arrest in all cell lines, increased senescence and cell death (except SKOV3).

Conclusions All analyzed cell lines, except SKOV3, had low olaparib IC50 values. The same correlation was found for the level of cell death and senescence what may be related to the functional status of BRCA1/2. Cell cycle G2/M phase arrest was observed in all ovarian cancer cell lines.

\section{REFERENCES}

1. Tudrej, P.et al.2018-10.3390/ijms19072080

2. Stordal, B.,et al.2013-10.1016/j.molonc.2012.12.007

A.J.C. was co-financed by the European Union through the European Social Fund (grant POWR.03.02.00-00-I029).

\section{IGCS20_1220}

\section{FUNCTIONAL ANALYSIS OF ZFHX4 AS A NOVEL THERAPEUTIC TARGET IN OVARIAN CANCER}

Y Oyama*, S Shigeta, K Tsuji, H Tokunaga, M Shimada, N Yaegashi. Tohoku University, Japan

10.1136/ijgc-2020-IGCS.188
Introduction Ovarian cancer is one of the most lethal gynecologic malignancies. Many patients are diagnosed in advanced stages of disease where the prognosis is poor due to the inevitable acquired resistance to platinum agents. Thus, novel therapeutic strategies effective for platinum-resistant cases are required. Zinc Finger Homeobox 4 (ZFHX4) has been known as one of the major transcription regulators in nerve and muscle differentiation. Recent researches including our genome-wide exploration indicate ZFHX4 is also involved in carcinogenesis and cancer progression. The objective of this study was to investigate the potential of ZFHX4 as a novel therapeutic target in ovarian cancer.

Methods Correlations between ZFHX4 mRNA expression and overall survival in 61 ovarian cancer cases treated at our institute and in the cases registered in TCGA database respectively were assessed by log-lank test. RNAi knockdown was introduced to several ovarian cancer cells to investigate the influence of ZFHX4 on cancer cell proliferation, migration and platinum sensitivity.

[Results] Both in our clinical dataset and TCGA data, the overall survival was significantly poor in the group in which ZFHX4 is highly expressed. In cell model assay, ZFHX4 knockdown significantly suppressed ovarian cancer cell proliferation. Decreased motility was observed in the cells treated with siRNA targeting ZFHX4 by scratch assay. Furthermore, ZFHX4 knockdown increased cisplatin sensitivity.

Conclusions Altogether, our results suggest ZFHX4 functions to promote cancer cell proliferation, migration and platinumresistance. Interruption of ZFHX4 function independently or in combination with platinum agents has a potential to bring benefit for the treatment of ovarian cancer.

\section{IGCS20_1221}

\section{PARAAORTIC LYMPHADENECTOMY IN CERVICAL CANCER STAGE IIIC1R INTERNATIONAL FEDERATION OF GYNECOLOGY AND OBSTETRICS 2018: EXPERIENCE AT THE INSTITUTO NACIONAL DE CANCEROLOGÍA}

1f Acosta*, ${ }^{2}$ J Rodriguez, ${ }^{3} \mathrm{~J}$ Velasquez, ${ }^{4} \mathrm{~L}$ Buitrago, ${ }^{5} \mathrm{O}$ Forero, ${ }^{6} \mathrm{~J}$ Peralta, ${ }^{1} \mathrm{~J}$ Saenz. ${ }^{1}$ Department of Gynecology Oncology. Instituto Nacional de Cancerología, Colombia; ${ }^{2}$ Department of Gynecology Oncology. Instituto Nacional de Cancerología. Department of Gynecology and obstetrics. Section of Gynecology Oncology. Fundación Santa Fe de Bogotá, Colombia; ${ }^{3}$ Department of Clinical Oncology. Instituto Nacional de Cancerología, Colombia; ${ }^{4}$ Department of statistics. Universidad Nacional de Colombia. Department of Epidemiology. Universidad de la Sabana, Colombia; ${ }^{5}$ Department of Radiology. Instituto Nacional de Cancerología, Colombia; ${ }^{6}$ Department of Gynecology and Obstetrics. Universidad Nacional de Colombia, Colombia

\subsection{6/ijgC-2020-IGCS. 189}

Introduction The objective of this study is to describe surgical and oncological outcomes of patients with cervical cancer stage IIIC1r FIGO 2018, underwent to staging paraaortic lymphadenectomy at the Instituto Nacional de Cancerología. Bogotá-Colombia, between January 2016 to March 2019.

Methods Retrospective study. Data collected included clinical characteristics, histopathological, surgical results. Furthermore, overall survival and disease-free survival were described in the population. A univariate descriptive analysis was performed. Survival curves were estimated with the KaplanMeier method. Statistical software R -Project version 3.6.2 was used. 
Results 40 patients were included in the analysis. The mean age was 43 years (29-66). Surgical approach was extraperitoneal in $31(77.5 \%)$ and transperitoneal in $9(22.5 \%)$ of the cases. The mean operating time was 147.5 minutes (120.2 -186.2). Surgical bleeding had a median of $30 \mathrm{ml}$ (10-50). There were no intraoperative complications or death associated with the procedure. The median paraaortic lymph node count was 8.5 nodes (5.75-15). Six (15\%) patients had para-aortic compromise and all received extended field radiotherapy. At follow-up, 5\% of patients presented recurrent disease $(n=1$ lung and $n=1$ supraclavicular lymph node). The overall survival (OS) was $88.7 \%$ and the disease-free survival (DFS) was $74.3 \%$ in the population.

Conclusion Paraaortic lymphadenectomy in stage IIIC1r cervical cancer in our study detected $15 \%$ of patients with lymph node involvement, without associated morbidity. It was not possible to evaluate the oncological impact of this procedure in the study population.

\section{IGCS20 1223}

\section{HIGH INCIDENCE OF VENOUS THROMBOEMBOLISM IN PATIENTS RECEIVING NEOADJUVANT CHEMOTHERAPY FOR ADVANCED STAGE OVARIAN CANCER}

S Chokshi* , J Gaughan, L Krill. Cooper University Hospital, USA

\subsection{6/ijgc-2020-IGCS.190}

Objectives There is paucity of data on venous thromboembolism (VTE) in patients receiving neoadjuvant chemotherapy (NACT) for ovarian cancer. We explored the incidence and predictors of VTE in this patient population.

Methods We performed a retrospective review of women with primary ovarian, fallopian tube or peritoneal cancer who received NACT from January 2012 to October 2018. Patients with a history of VTE prior to cancer diagnosis were excluded. The primary outcome was incidence of deep vein thrombosis (DVT) or pulmonary embolism (PE) after cancer diagnosis and before interval debulking surgery. We explored demographic and clinical variables associated with VTE.

Results VTE was diagnosed in 25 (28\%) of 90 patients and 16 (64\%) had PE. 67\% of patients had VTE during NACT and 8 patients after their cancer diagnosis, before initiation of NACT. The majority of patients had stage III disease and serous adenocarcinoma. African Americans were 3 times more likely than other races to experience VTE (OR 3.22; CI 0.997-10.42; P = 0.051). Significantly more patients without VTE had debulking surgery $(88 \%$ vs $60 \%$, $\mathrm{P}=0.005)$. The risk of DVT increased by $8.7 \%$ per year of age (OR 1.087; 95\% CI 1.01-1.17). Obesity, smoking status, medical comorbidities, disease stage, histology, invasive diagnostic surgery, and length of NACT were not predictors of VTE.

Conclusions The incidence of VTE during neoadjuvant chemotherapy is high. Older age and African American race may be potential risk factors for VTE. This information will help mitigate disparities in the treatment of advanced stage ovarian cancer.
IGCS20_1224

\section{A THREE PROTEIN SIGNATURE FAILS TO EXTERNALLY VALIDATE AS A BIOMARKER TO PREDICT SURGICAL OUTCOME IN HIGH GRADE SEROUS OVARIAN CANCER}

${ }^{1} \mathrm{~A}$ Hawarden* ${ }^{*}{ }^{2} \mathrm{M}$ Price, ${ }^{3} \mathrm{G}$ Wilson, ${ }^{2} \mathrm{~B}$ Russell, ${ }^{4} \mathrm{~L}$ Farrelly, ${ }^{5} \mathrm{~A}$ Embleton-Thursk, ${ }^{5} \mathrm{M}$ Parmar, ${ }^{1} \mathrm{RJ}$ Edmondson. ${ }^{1}$ Faculty of Biology, Medicine and Health, University of Manchester and Department of Gynaecological Oncology surgery, Saint Marys Hospital, Manchester University NHS Trust, UK; ${ }^{2}$ Faculty of Biology, Medicine and Health, University of Manchester, UK; ${ }^{3}$ Department of Pathology, Manchester University NHS Foundation Trust, UK; ${ }^{4}$ Cancer Research UK and University College London Cancer Trials Centre, UK: ${ }^{5}$ MRC Clinical trials Unit, University College London, UK

\subsection{6/ijgc-2020-IGCS. 191}

Introduction Complete cytoreduction is associated with improved survival in patients with advanced High Grade Serous Ovarian Cancer (HGSOC). To aid clinical decision making, many surgical outcome prediction tools have been proposed, but none have been sufficiently validated to warrant routine clinical usage. Here we attempted to externally validate a promising three protein signature, which had shown strong association with suboptimal surgical debulking (AUC 0.89 , accuracy $92.8 \%$ ).

Methods 241 HGSOC tumour samples were collected from patients who participated in a large multicentre trial (ICON5). Samples were collected at the time of initial surgery and before randomisation. Surgical outcome data were collated from the study records. Immunohistochemical scores were generated by two independent observers for the three proteins in the original signature (POSTN, CXCL14 and pSmad2/3). Predictive values were generated for individual and combination protein signatures and as part of a multivariable model using logistic regression.

Results When assessed individually, none of the proteins showed any predictive affinity for suboptimal surgical outcome in our cohort (AUC POSTN 0.55, pSmad 2/3 0.53, CXCL 14 0.62 ). The combined signature again showed poor predictive ability, AUC 0.58, as did the multivariable model, AUC 0.63. Conclusion Despite showing original promise, when this protein signature is applied to a large external cohort, it is unable to accurately predictive surgical outcomes. This could be attributed to overfitting of the original model, or differences in surgical practice in our cohort.

\section{IGCS20_1225}

\section{ADJUVANT CHEMOTHERAPY IN SURGICAL STAGE I OR II ENDOMETRIOID ENDOMETRIAL CANCER WITH MYOMETRIAL INVASION > 50\%: A MULTICENTER RETROSPECTIVE STUDY WITH LONG-TERM FOLLOW-UP}

${ }^{1} \mathrm{~S}$ Garzon*, ${ }^{2} \mathrm{~F}$ Multinu, ${ }^{3} \mathrm{~A}$ Weaver, ${ }^{3} \mathrm{ME}$ McGree, ${ }^{4} \mathrm{E}$ Sartori, ${ }^{5} \mathrm{~F}$ Landoni, ${ }^{6} \mathrm{P}$ Zola, ${ }^{1} \mathrm{G}$ Dinoi, ${ }^{2} \mathrm{G}$ Aletti, ${ }^{7} \mathrm{~A}$ Gadducci, ${ }^{1} \mathrm{~A}$ Mariani. ${ }^{1}$ Department of Gynecology and Obstetrics, Mayo Clinic, USA; ${ }^{2}$ Division of Gynecologic Oncology, IEO, European Institute of Oncology IRCCS, Italy; ${ }^{3}$ Division of Biomedical Statistics and Informatics, Mayo Clinic, USA; ${ }^{4}$ Department of Gynecology and Obstetrics, University of Brescia, Italy; ${ }^{5}$ Department of Medicine and Surgery, Clinic of Obstetrics and Gynecology, San Gerardo Hospital Monza, University of Milan Bicocca, Italy; ${ }^{6}$ Department of Surgical Sciences, University of Turin, Italy; ${ }^{7}$ Department of Medical Oncology, Mayo Clinic, USA

\subsection{6/ijgc-2020-IGCS. 192}

